\title{
Rituximab, Cyclophosphamide, and Corticosteroids for ANCA Vasculitis: The Good, the Bad, and the Ugly
}

\author{
Roberta Fenoglio Dario Roccatello Savino Sciascia \\ Nephrology and Dialysis Unit \& CMID (Center of Research of Immunopathology and \\ Rare Diseases), Coordinating Center of the Network for Rare Diseases of Piedmont and \\ Aosta Valley, San Giovanni Bosco Hub Hospital of Turin and Department of Clinical and \\ Biological Sciences, University of Turin, Turin, Italy
}

\section{Keywords}

Rituximab · Cyclophosphamide · Corticosteroids - ANCA vasculitis

\begin{abstract}
Backgrounds: ANCA-associated vasculitis (AAV) frequently present with a chronic relapsing course. Relapse leads to an increased need for therapeutic agents and consequent toxicity. Summary: When referring to the available options for the management of AAV, the efficacy of glucocorticoids (GCs) is unquestionable. However, similarly unquestionable are their side effects. It has been more than 40 years since the efficacy of cyclophosphamide (CYC) as an add-on therapy to GCs in the management of necrotizing vasculitis has been proven. At the same time, concerns about the devastating side effects related to a prolonged exposure to this agent were raised. Despite the well-known side effects, the management of AAV remained centred on CYC until the early 2000s, when the pilot data first supporting the anecdotal efficacy of rituximab (RTX) were reported. However, it was not until 2010 that the noninferiority of RTX to CYC for remission-induction in AAVs was demonstrated in 2 randomized controlled trials. Key Messages: Treatment of AAV has improved over the last decade, and currently available strategies are able to induce remission in the majority of the cases. Herewith, we aim to critically review available evidence and to critically address the following question: How can we reduce the GCs use the management of patients with AAV? Novel strategies that avoid the toxicity associated with currently used agents should be the goal. Ideally, these approaches should be GC-free.




\section{Introduction}

ANCA-associated vasculitis (AAV) frequently present with a chronic relapsing course. Relapse leads to an increased need for therapeutic agents and consequent toxicity. When referring to the available options for the management of AAV, the efficacy of glucocorticoids (GCs) is unquestionable. However, similarly unquestionable are their side effects.

It has been more than 40 years since Fauci et al. [1] proved the efficacy of cyclophosphamide (CYC) as an add-on therapy to GCs in the management of necrotizing vasculitis. While they demonstrated the efficacy of the CYC in addition to GCs in inducing remission also in the most severe cases, at the same time, concerns about the devastating side effects related to a prolonged exposure to this protocol were raised. Since then, a tremendous body of research has been conducted to find alternative agents to GCs for the management of AAV. In regards to the use of immunosuppressants, the research efforts were aiming at optimizing available protocols, to decrease the cumulative dose of cytotoxic agents such as CYC, and to guide the switching from an oral to an intravenous pulsed to reduce prolonged toxicity [2]. However, the constant unmet need was to identify protocols with a satisfactory efficacy and safety profile paralleled by a steroid-sparing effect and incorporate them in remission-maintenance regimens in order to taper GCs more rapidly while at the same time reduce the risk of relapse.

Despite the well-known side effects, the management of AAV remained centred on CYC until the early 2000s, when the pilot data first supporting the anecdotal efficacy of rituximab (RTX) were reported [3]. However, it was not until 2010 that the non-inferiority of RTX to CYC for remission-induction in AAVs was demonstrated in 2 randomized controlled trials (RCTs) (RAVE [4] and RITUXVAS [5]). Nevertheless, although the 2 RCTs have demonstrated RTX to be the most effective induction therapy in patients with relapsing disease, the optimal treatment duration and RTX dose are still discussed. Similarly, the debate whether to administer a maintenance dose to every patient, at a fixed time interval or on the basis of B-cell count and ANCA titre or only when disease manifestations do occur is currently ongoing.

Two subsequent RCTs (MAINRITSAN [6] and MAINRITSAN2 [7]) investigated the efficacy of RTX in remission maintenance. The main characteristics of the RTCs investigating the use of RTX are summarized in Table 1. The MAINRITSAN study showed a superiority of RTX given in 6 fixed-schedule monthly infusion over azathioprine (AZA) in reducing the rate of major relapse. A further benefit of RTX was also observed when analysing physical abilities and quality of life [8]. Conversely, an individually tailored RTX administration was investigated in the MAINRITSAN2. When monitoring patients every 3 months, cases were given RTX in the presence of disease reactivation, namely, peripheral B-cell repopulation or the increase of ANCA titres. Controls received a fixed $500 \mathrm{mg}$ RTX on a pre-fixed protocol on days 0 and 14 post-randomization, then 6,12 , and 18 months after the first infusion. The main findings of this study rely on the observation that relapse rates did not differ significantly between individually tailored and fixed-schedule RTX regimens. However, patients in individually tailoredarm received fewer RTX infusions. The MAINRITSAN3 is currently ongoing (clinicaltrials.gov \#NCT02433522). The investigators aim to conduct a randomized placebo-controlled trial of a long-term RTX maintenance treatment (46 months) against a conventional maintenance treatment (18 months).

The recruitment of a further study, the RITAZAREM (clinicaltrials.gov \#NCT01697267), has been recently listed as completed and results are highly expected (clinicaltrials.gov \#NCT02433522). This RTCs recruited AAV patients at the time of relapse, all receiving 4 weekly infusion of RTX at the dose of $375 \mathrm{mg} / \mathrm{m}^{2}$ and GCs. After 4 months, in case remission is achieved, further treatment with RTX (a single dose over 4 months for 2 years) or AZA will be chosen randomly. The patients will be followed for 4 years. 
Table 1. Main characteristics of randomized controlled trials investigating the use of RTX in AAV

\begin{tabular}{|c|c|c|}
\hline Study & Protocol & GCs \\
\hline RAVE [4] & $\begin{array}{l}\text { RTX group: } \\
\text { RTX } 375 \mathrm{mg} / \mathrm{m}^{2} \text { once weekly for } 4 \text { weeks plus daily } \\
\text { placebo-CYC. } \\
\text { Control group: } \\
\text { Placebo-RTX infusions plus daily CYC }(2 \mathrm{mg} / \mathrm{kg} \text {, adjusted } \\
\text { for renal insufficiency) }\end{array}$ & $\begin{array}{l}\text { One to } 3 \text { pulses of MP ( } 1,000 \mathrm{mg} \text { each }) \text {, followed by } \\
\mathrm{P} \text { at a dose of } 1 \mathrm{mg} / \mathrm{kg} / \text { day. The dose was tapered so } \\
\text { that by } 5 \text { months, all patients who had a remission } \\
\text { without disease flares had discontinued GCs }\end{array}$ \\
\hline RITUXIVAS [5] & $\begin{array}{l}\text { RTX group: } \\
\text { RTX } 375 \mathrm{mg} / \mathrm{m}^{2} \mathrm{IV} \times 4 \text { once weekly for } 4 \text { weeks plus CYC } \\
15 \mathrm{mg} / \mathrm{kg} \mathrm{IV} \times 2 \\
\text { CYC group: } \\
\text { IV CYC } 15 \mathrm{mg} / \mathrm{kg} \mathrm{IV} \times 2 \text { (minimum } 3 \text { months, maximum } \\
6 \text { months) }\end{array}$ & $\begin{array}{l}\text { IV MP (at a dose of } 1 \mathrm{~g} \text { ) followed by P }(1 \mathrm{mg} / \mathrm{kg} / \text { day } \\
\text { initially, with a reduction to } 5 \mathrm{mg} / \text { day at the end of } \\
6 \text { months) }\end{array}$ \\
\hline MAIN 1 [6] & $\begin{array}{l}R T X(\operatorname{arm} A) \text { : } \\
\text { RTX infusion will be performed at D1, D15, M6, M12, and } \\
\text { M18 (i.e., a total of } 5 \text { infusions), at the dose of } 500 \mathrm{mg} \text { at } \\
\text { a fixed dosage } \\
\text { AZA ( } \operatorname{arm} B): \\
\text { AZA }(2 \mathrm{mg} / \mathrm{kg} / \text { day) for } 12 \text { months, then progressively } \\
\text { tapered until its discontinuation at month } 22\end{array}$ & $\begin{array}{l}\mathrm{P} \text { (or equivalent) at a dose of } 1 \mathrm{mg} / \mathrm{kg} / \text { day with } \\
\text { gradual tapering according to a regimen adjusted to } \\
\text { body weight over a mean of } 18 \text { months since } \\
\text { diagnosis }\end{array}$ \\
\hline MAIN 2 [7] & $\begin{array}{l}\text { RTX }(\operatorname{arm} A) \text { : } \\
\text { RTX infusion at D1, D15, M6, M12, and M18 (i.e., a total of } \\
5 \text { infusions), at the dose of } 500 \mathrm{mg} \text { at a fixed dosage } \\
\text { RTX }(\operatorname{arm} B) \text { : } \\
\text { RTX infusion at D1 and then ANCA status and CD19+ } \\
\text { lymphocyte count monitored every } 3 \text { months. Patients } \\
\text { received new } 500 \text { mg RTX infusions either if CD19 were } \\
>\text { to } 0 / \mathrm{mm}^{3} \text { or if ANCA are positive again or if ANCA titre } \\
\text { significantly raised }\end{array}$ & $\begin{array}{l}\mathrm{P} \text { (or equivalent) } 1 \mathrm{mg} / \mathrm{kg} / \text { day with gradual } \\
\text { tapering according to a regimen adjusted to body } \\
\text { weight over a mean of } 18 \text { months since diagnosis }\end{array}$ \\
\hline
\end{tabular}

AAV, ANCA-associated vasculitis; GCs, glucocorticoid; RTX, rituximab; CYC, cyclophosphamide; MP, methylprednisolone; P, prednisone; AZA, azathioprine; PTS, patients; D, day; M, month.

Several other regimens exploring the use of RTX as a maintenance agent have explored over the last years, to include protocol with low-dose RTX in patients with limited forms of granulomatosis with polyangiitis [9]. When taken together the above mentioned data, while the efficacy of RTX seems consistent across the studies, a main question remains unanswered: How can we reduce the GCs use the management of patients with AAV?

In fact, patients included in the main RCTs to investigate the efficacy of RTX were exposed to similar overall doses of GCs. Furthermore, reducing the use GCs especially in the maintenance phase might pose some concerns in the treating physicians.

The REMAIN trial [10] was a prospective randomized trial to compare 2 different durations of maintenance immunosuppressive therapy for the prevention of relapse in AAV. Patients with AAV who were in stable remission after CYC/GCs-based induction followed by AZA/GCs maintenance therapy were randomized (1:1) to receive continued AZA/GCs for 48 months (continuation group) or to withdraw AZA/GCs by 24 months (withdrawal group). They found a 2-fold increase in the risk of relapse in the withdrawal group and a reduced renal survival in AAV when compared to the continuation group.

The CLEAR trial pioneered the search for GC-free regimen in the management of AAV. They aimed at investigating if avacopan (CCX168), an orally administered, selective C5a receptor inhibitor, could replace oral GCs without impacting efficacy. Newly diagnosed or 
relapsing AAV patients were randomized to received placebo plus prednisone starting at 60 $\mathrm{mg}$ daily (control group), avacopan (30 mg, twice daily) plus reduced-dose prednisone (20 $\mathrm{mg}$ daily), or avacopan (30 mg, twice daily) without prednisone. All patients received CYC or RTX. With a primary efficacy end point of treatment response at week 12 (defined as a BVAS decrease from baseline of at least $50 \%$ plus no worsening in any body system), they concluded that avacopan was effective in replacing high-dose GCs in the management of AAV. A doubleblind, active-controlled, phase 3 trial is currently ongoing [11].

More recently, a reduced-dose GCs regimen was tested in patients with end-organ damage in the lately published PEXIVAS [12] trial. The study sought to determine whether patients with severe renal impairment and/or pulmonary haemorrhage at disease onset benefited from the addition of plasmapheresis to a standard remission-induction regimen (as was suggested by the MEPEX [13] trial) either with a standard or reduced-dose GCs regimen. While the study showed that plasmapheresis did not add any benefit in terms of risk reduction of hard outcomes (death or ESRD), the reduced-dose GCs arm achieved the same efficacy as the standard-dose arm with fewer serious infections.

In order to participated in this debate, we have previously reported the encouraging results of 11 patients with refractory AAV treated with a " $4+2$ " RTX protocol $[14,15]$. In $81 \%$ of the patients, RTX was given due to lack of response or occurrence of side effects after treatment with CYC. The " $4+2$ " protocol (so called improved protocol $[14,15]$ ) showed a good safety profile and its efficacy was confirmed also in a long-term observational study. In detail, after a mean follow-up of 85 months since the " $4+2$ " RTX protocol, $37 \%$ of the patients (1 eosinophilic granulomatosis with polyangiitis and 3 microscopic polyangiitis, all MPOpositive) achieved persistent remission after 1 cycle of " $4+2$ " RTX protocol with no further relapse observed after up to 108 months. After a median time of more 4 years, 7 out of 11 patients suffered from a relapse and they were re-treated with RTX (again as monotherapy with the same protocol). Following reinduction, they again achieved a complete response which was maintained up to 96 months of observation. The strengths of the " $4+2$ " approach in the management of the most severe case/refractory cases of AAV relies on the sustained clinical remission without immunosuppressive maintenance therapy and a negligible dose of prednisone since the 5 th months.

We recently introduced a therapeutic algorithm combing both clinical and histological features to guide AAV management. On the basis of our previous positive experience in patients with severe lupus nephritis [16], in AAV patients with serum $\mathrm{Cr}$ (sCr) levels higher than $5 \mathrm{mg} / \mathrm{dL}$ and $>50 \%$ epithelial (florid) crescents at the renal biopsy (so called "crescentic forms") the " $4+2$ " RTX protocol was implemented with 2 pulse ( 2 weeks apart) of $15 \mathrm{mg} / \mathrm{kg}$ CYC, adjusted for the renal impairment, in order to potentiate the CD20+ B-cells depletion. This intensive B-cell depletion therapy (IBCDT) protocol has been already applied to 12 severe patients with microscopic polyangiitis (mean $\mathrm{sCr} 5.8 \mathrm{mg} / \mathrm{dL}$ ). After a mean observation time of $>1$ year, 8 patients $(3$ with $>50 \%$ and 5 with $<50 \%$ florid crescents, including 1 case having $>50 \%$ glomerular sclerosis) were haemodialysis-free and in clinical remission. All 4 patients with granulomatosis with polyangiitis achieved a complete response of with resolution of systemic symptoms.

Histological features of striking renal response obtained with the RTX-based protocols in our cohort and clinical points of interest are highlighted in Figures 1 and 2. Subjects have given their written informed consent to publish their case. Data collection has been conducted according to the Italian regulation on rare diseases. 

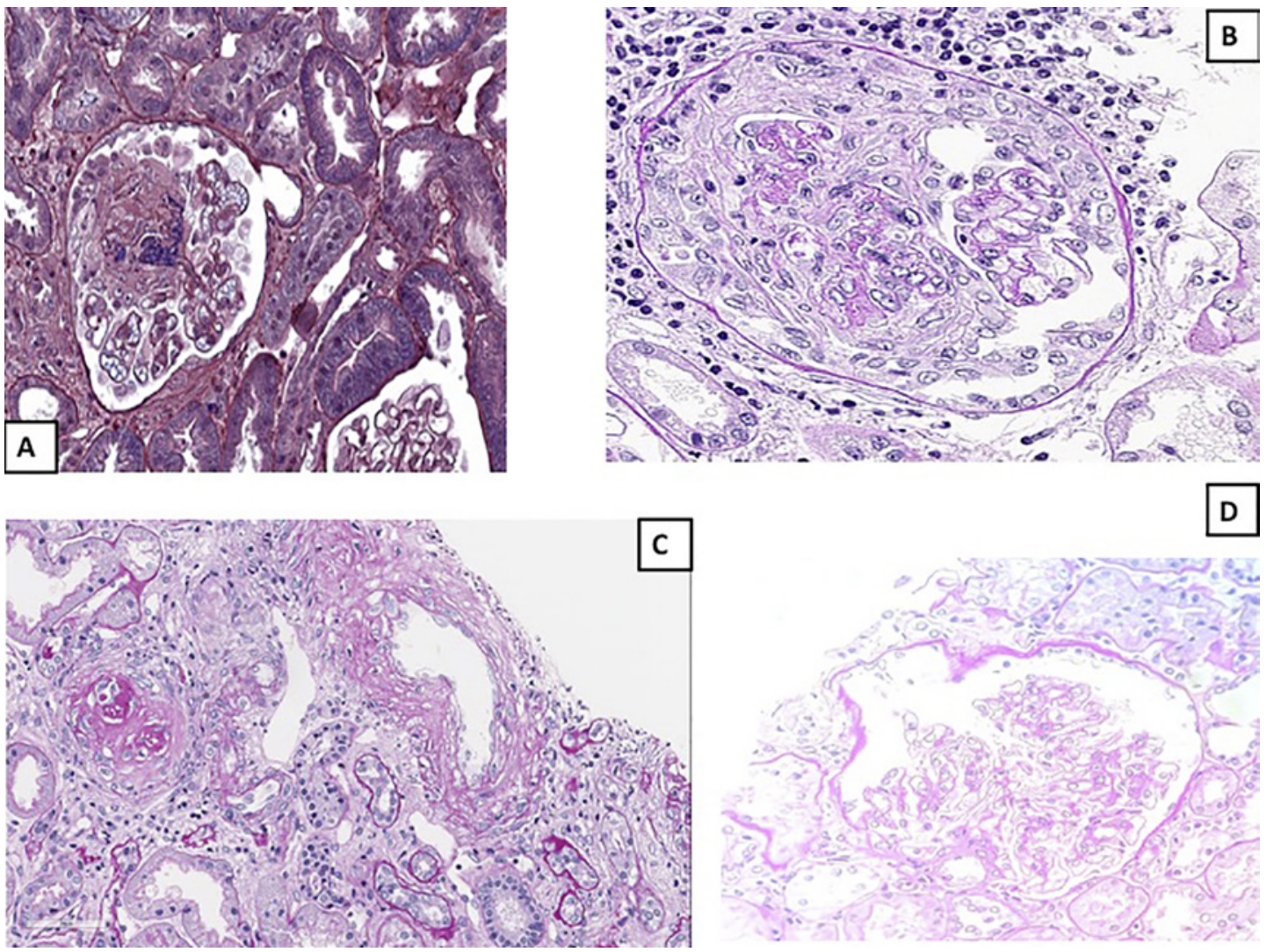

Fig. 1. The picture shows a glomerulus with segmental epithelial crescent with an area of fibrinoid necrosis (A) and a glomerulus with circumferential epithelial crescent (B). On her second biopsy and after treatment with IBCDT, we found only mild arterial intimal fibrosis (C), while glomeruli had no signs of crescent formation in Bowman's space (D). IBCDT, intensive B-cell depletion therapy.

\section{Case 1}

A 58-year-old woman had a renal biopsy due to sub-nephrotic proteinuria and microscopic haematuria combined with elevated cANCA levels and bilateral lung thickenings. Biopsy revealed a necrotizing vasculitis with associated endo- and extra-proliferative glomerulonephritis. The picture shows a glomerulus with segmental epithelial crescent with an area of fibrinoid necrosis (Fig. 1A), and a glomerulus with circumferential epithelial crescent (Fig. 1B). The patient was given corticosteroids and oral CYC for 6 months followed by AZA for 3 years with resolution of urinary abnormalities and lung lesions, and serologic normalization. Nine years later, the patient had a renal relapse with sub-nephrotic proteinuria and renal impairment (with serum $\mathrm{Cr}$ of $2.4 \mathrm{mg} / \mathrm{dL}$ ) and new cANCA elevation. She was administered a single cycle of IBCDT consisting of RTX $375 \mathrm{mg} / \mathrm{sm}$ given weekly 4 times with 2 more infusions 1 and 2 months later, combined with 2 infusions of $10 \mathrm{mg} / \mathrm{kg}$ of cyclophosphamide, and 3 pulses of $1 \mathrm{~g}$ of methylprednisolone followed by prednisone 0.8 $\mathrm{mg} / \mathrm{kg}$ tapered to $5 \mathrm{mg}$ in 3 months. The IBCDT achieved a disappearance of proteinuria and a decrease in serum $\mathrm{Cr}$ to $1.5 \mathrm{mg} / \mathrm{dL}$. No further immunosuppressive therapy was administered. Five years later, the patient presented with $1 \mathrm{~g} /$ day proteinuria, increased inflammation parameters and cANCA titre. The possibility of a new relapse was investigated with a new biopsy. Apart from global sclerosis in 30\% glomeruli, and mild signs of arteriolosclerosis, renal parenchyma was found to be substantially normal. The picture 

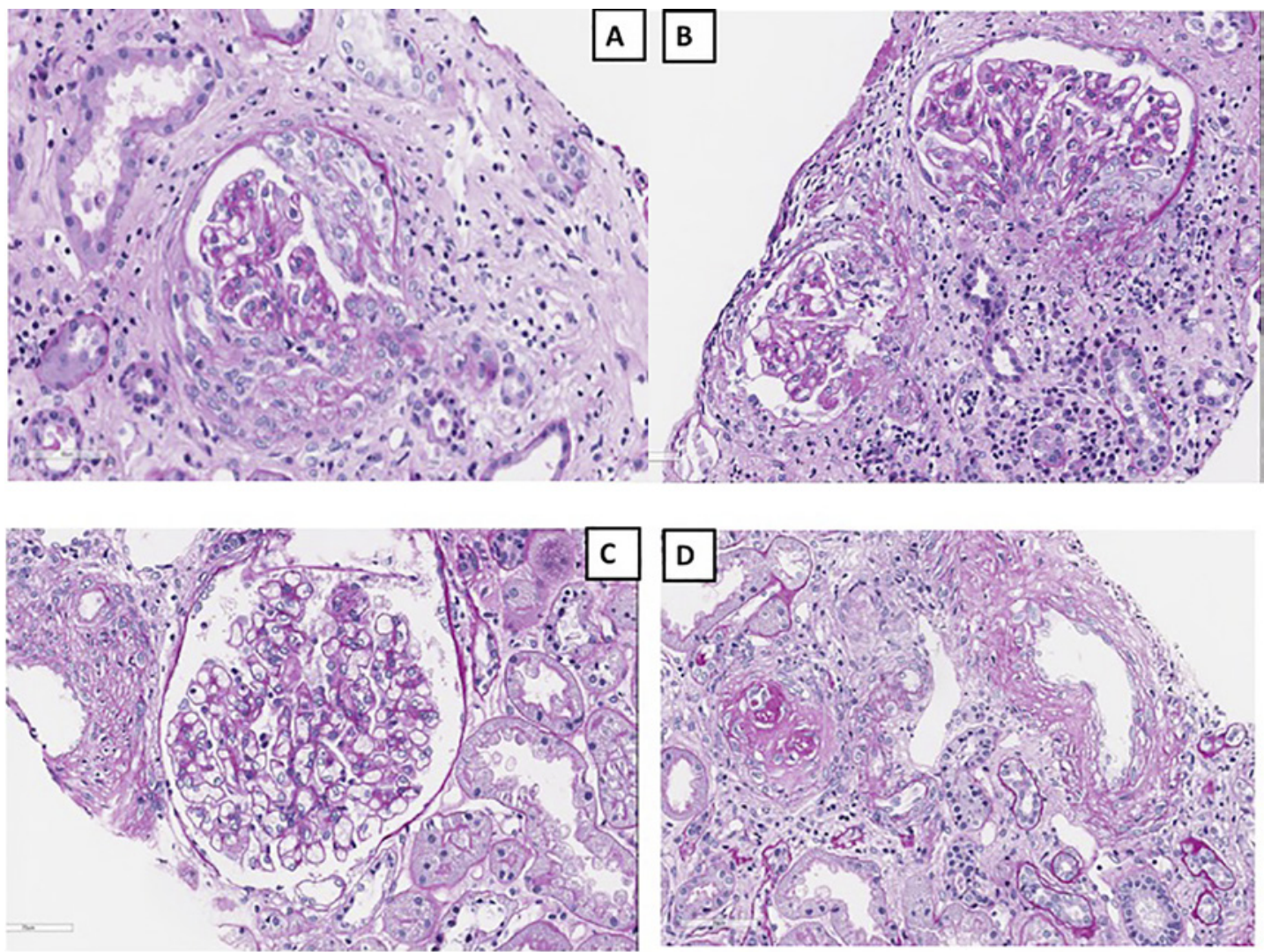

Fig. 2. The picture shows a glomerulus with a florid circumferential crescent (A), and an extensive lymphocyte interstitial infiltrate surrounding crescentic glomeruli (B). The second biopsy revealed 20\% of global glomerular sclerosis without signs of proliferation. The picture shows a normal glomerulus (C) and signs of interstitial fibrosis with a sclerotic glomerulus (D).

shows only mild arterial intimal fibrosis (Fig. 1C), while glomeruli had no signs of crescent formation in Bowman's space (Fig. 1D).

The patient was given GCs and oral CYC for 6 months followed by AZA for 3 years with resolution of urinary abnormalities and lung lesions and serologic normalization. Nine years after the diagnosis, the patient suffered from a renal relapse with sub-nephrotic proteinuria and renal functional impairment. She was administered a single cycle of the IBCDT followed by prednisone $0.8 \mathrm{mg} / \mathrm{kg}$ tapered to $5 \mathrm{mg}$ in 3 months. This case emphasizes the critical role of renal biopsy in discriminating between a renal relapse and the appearance of proteinuria as a consequence of glomerular sclerosis and parenchymal adaptation following resolution of the inflammatory process.

\section{Case 2}

This is a 62-year-old male with a necrotizing pauci immune glomerulonephritis with $>50 \%$ of crescents, presenting with acute kidney injury (serum $\mathrm{Cr} 9 \mathrm{mg} / \mathrm{dL}$ and oliguria), mild pulmonary thickenings, extremely high anti-MPO ANCAlevels, a severe peripheral neuropathy, and BVAS score 23. He was successfully treated with the IBCDT. The picture shows a glomerulus with a florid circumferential crescent (Fig. 2A) and an extensive lymphocyte inter- 
stitial infiltrate surrounding crescentic glomeruli (Fig. 2B). In order to define the need of a maintenance immunosuppressive therapy, the patient was re-biopsied 6 months later when presenting with $1.5 \mathrm{mg} / \mathrm{dL}$ of serum $\mathrm{Cr},<0.5 \mathrm{~g}$ /day proteinuria, mild lung interstitial fibrosis, and remarkable improvement of electromyography. BVAS score was 3 . The biopsy revealed $20 \%$ of global glomerular sclerosis without signs of proliferation. The picture shows a normal glomerulus (Fig. 2C), and signs of interstitial fibrosis with a sclerotic glomerulus (Fig. 2D).

This case again emphasizes the role of renal biopsy in the management of ANCA vasculitis patients. Renal biopsy may be decisive in doubtful cases, and it is likely to be much less risky than an unneeded immunosuppressive therapy (especially if prolonged like a remission maintenance).

What can we learn from these cases? While reducing the exposure to toxic drugs and limiting the use of GCs remain among the main unmet needs in the field of AAV, one cannot forget that the identification of novel biomarkers or prognostic factors is still warranted. More critically, integrating biomarkers in the therapeutic strategies will pave the way for tailored treatment. When focussing on risk factors for relapses, for instance, several studies have demonstrated that the relapse rate is higher in patients with PR3-AAV when compared to MPO-AAV. Similarly, PR3-ANCA has been reported as an independent risk factor for renal outcomes in various analyses, albeit some degree of discrepancy among prospective and retrospective data still exists [17-19]. In our previously mentioned long-term experience, we observed that all 4 patients relapsing in the first 5 years of follow-up after the first " $4+2$ " RTX cycle were PR3 positive.

\section{Conclusion}

Treatment of AAV has improved over the last decade, and currently available strategies are able to induce remission in the majority of the cases. Novel strategies that avoid the toxicity associated with currently used agents should be the goal. Ideally, these approaches should be GCs free. As the AAV are typically characterized by a relapsing course, and in order to optimize tailored approaches for the affected patients, physicians should be aware of unique features that may influence clinical response.

\section{Statement of Ethics}

Subjects have given their written informed consent to publish their case (including publication of images from renal biopsy). Data collection has been conducted according to the Italian regulation on rare diseases.

\section{Conflict of Interest Statement}

The authors have no conflicts of interest to declare.

\section{Funding Sources}

This manuscript was not supported by any specific funding source. 


\section{Author Contributions}

R.F., D.R., and S.S. drafted the manuscript, designed the layout, reviewed the literature, and critically edited the manuscript.

\section{References}

1 Fauci A, Katz P, Haynes B, Wolff S. Cyclophosphamide therapy of severe systemic necrotizing vasculitis. N Engl J Med. 1979;301(5):235-8.

2 De Groot K, Harper L, Jayne D, Flores Suarez LF, Gregorini G, Gross WL, et al. Pulse versus daily oral cyclophosphamide for induction of remission in antineutrophil cytoplasmic antibody-associated vasculitis: a randomized trial. Ann Intern Med. 2009;150(10):670-80.

3 Specks U, Fervenza FC, Mcdonald TJ, Hogan MC. Response of Wegener's granulomatosis to anti-CD20 chimeric monoclonal antibody therapy. Arthritis Rheum. 2001;44(12):2836-40.

4 Stone J, Merkel P, Spiera R, Seo P, Langford CA, Hoffman GS, et al. Rituximab versus cyclophosphamide for ANCA-associated vasculitis. N Engl J Med. 2010;363(3):221-32.

5 Jones R, Tervaert JW, Hauser T, Luqmani R, Morgan MD, Peh CA, et al. Rituximab versus cyclophosphamide in ANCA-associated renal vasculitis. N Engl J Med. 2010;363(3):211-20.

6 Guillevin L, Pagnoux C, Karras A, Khouatra C, Aumaître O, Cohen P, et al. Rituximab versus azathioprine for maintenance in ANCA-associated vasculitis. N Engl J Med. 2014;371(19):1771-80.

7 Charles P, Terrier B, Perrodeau E, P, S, A, et al. Comparison of individually tailored versus fixed-schedule rituximab regimen to maintain ANCA-associated vasculitis remission: results of a multicentre, randomized controlled, phase III trial (MAINRITSAN2). Ann Rheum Dis. 2018;77(8):1143-49.

8 Pugnet G, Pagnoux C, Terrier B, Perrodeau E, Puéchal X, Karras A, et al. Rituximab versus azathioprine for ANCAassociated vasculitis maintenance therapy: impact on global disability and health-related quality of life. Clin Exp Rheumatol. 2016;34:S54-9.

9 Puechal X, Iudici M, Calich AL, Vivot A, Terrier B, Régent A, et al. Rituximab for induction and maintenance therapy of granulomatosis with polyangiitis: a single-centre cohort study on 114 patients. Rheumatology. 2019;58:401-9.

10 Karras A, Pagnoux C, Haubitz M, Groot K, Puechal X, Tervaert JWC, et al. Randomised controlled trial of prolonged treatment in the remission phase of ANCA-associated vasculitis. Ann Rheum Dis. 2017;76(10): 1662-8.

11 Jayne D, An B, Harper L, Schaier M, Venning M, Hamilton P, et al. Randomized trial of C5a receptor inhibitor avacopan in ANCA-associated vasculitis. J Am Soc Nephrol. 2017;28:2756-67.

12 Merkel PA, Jayne DR, Wang C, Hillson J, Bekker P. Evaluation of the safety and efficacy of avacopan, a C5a receptor inhibitor, in patients with antineutrophil cytoplasmic antibody-associated vasculitis treated concomitantly with rituximab or cyclophosphamide/azathioprine: protocol for a randomized, double-blind, activecontrolled, phase 3 trial. JMIR Res Protoc. 2020 Apr 7;9(4):e16664.

13 Jayne D, Gaskin G, Rasmussen N, Abramowicz D, Ferrario F, Guillevin L, et al. Randomized trial of plasma exchange or high-dosage methylprednisolone as adjunctive therapy for severe renal vasculitis. J Am Soc Nephrol. 2007;18(7):2180-8.

14 Roccatello D, Sciascia S, Rossi D, Alpa M, Naretto C, Radin M, et al. The "4 plus 2" rituximab protocol makes maintenance treatment unneeded in patients with refractory ANCA-associated vasculitis: a 10 years observation study. Oncotarget. 2017 May 23;8(32):52072-7.

15 Roccatello D. "How i treat" autoimmune diseases: state of the art on the management of rare rheumatic diseases and ANCA-associated systemic idiopathic vasculitis. Autoimmun Rev. 2017;16(10):995-8.

16 Roccatello D, Sciascia S, Baldovino S, Rossi D, Alpa M, Naretto C, et al. A 4-year observation in lupus nephritis patients treated with an intensified B-lymphocyte depletion without immunosuppressive maintenance treatment-Clinical response compared to literature and immunological re-assessment. Autoimmun Rev. 2015 Dec;14(12):1123-30.

17 Puéchal X, Pagnoux C, Perrodeau É, Hamidou M, Boffa J-J, Kyndt X, et al. Long-term outcomes among participants in the WEGENT trial of remission-maintenance therapy for granulomatosis with polyangiitis (Wegener's) or microscopic polyangiitis. Arthritis Rheumatol. 2016;68:690-701.

18 Slot MC, Tervaert JW, Boomsma MM, Stegeman CA. Positive classic antineutrophil cytoplasmic antibody (C-ANCA) titer at switch to azathioprine therapy associated with relapse in proteinase 3-related vasculitis. Arthritis Rheum. 2004;51(2):269-73.

19 Cartin-Ceba R, Golbin JM, Keogh KA, Peikert T, Sánchez- Menéndez M, Ytterberg SR, et al. Rituximab for remission induction and maintenance in refractory granulomatosis with polyangiitis (Wegener's): ten-year experience at a single center. Arthritis Rheum. 2012;64:3770-8. 